\title{
BIBLIOGRAFIE
}

\section{SEPARATE PUBLICATIONS}

(3I)

Continued from Nieuwe W. I. Gids 40, p. 171-183. - Publications not seen by the compiler of this incomplete bibliography are not included. The existence of a summary is indicated by a translation of the title.

Aafjes, Bertus: Maria Sybilla Merian, $4^{\text {th }}$ impr. J. M. Meulenhof, De Ceder, 1956, 3I pp., 3+ I (cover) col. pl. excl.

Poetry. - Ist edn. 1946; 2nd 1947; 3rd 1952.

Addresses to the Opening Session $5^{\text {th }}$ Inter-Guiana Geological Conference. Georgetown 1959, II pp.

AdHIN, Jan Hansdew: Development planning in Surinam in historical perspective (with special reference to the Ten Year Plan). Diss. Groningen 26. VI. 1961, xii +215 pp., 3 graphs.

Ahlbrinck CssR, W. \& Donicie CssR, A.: Ondrofeni sa leri ju. Vertellingen in het Neger-Engels met vertaling in het Nederlands. Tori's II. Varekamp \& Co. Paramaribo, dd. Oct. I958, 73 pp., ill. cover.

All Colors Co-Operation. The Voice of European Overseas Territories. Uitgave 'Abuwa' - Europe's America. West-Indians - Africans and Asian-Voice. Headoffice: Cooperation for Intercontinental Social Economic Development. Editor-Dir.: H. FAVERUs. Eerste Jaargang no. 4, I 8 pp. $27 \times 20 \mathrm{~cm}$, ill. (I960?); vol. 5, 6, 7, 8, 9 and (leaflet) ro. Special issue 1960,22 pp. $37 \times 27 \mathrm{~cm}$.

Alle Hens, Maandblad der Koninklijke Marine, Aug. 1961, Suriname en de Nederlandse Antillen, p. I-2I, 29 figs. - Text and ill. adapted from Marineblad, Dec. I96o.

Almanaque de Curaçao para el año de I96r. Compilación de W. M. Hoyer Succ. Ed. 4I. Curaçao, (r960), roo pp., portr.

AMson, F. W. vAN : Bodemonderzoek naar de oorzaken van groeiverschillen bij cacao op de plantages De Maasstroom en Berlijn. Meded. Landbouwproefsta. Sur. 23, I96I, 44 Pp. (22) figs.

A.N.: El unico religion berdadero. Uitgave: Boekcentrale Aquinas, Curaçao, 40 pp. I6 $\frac{1}{2} \times 1 I_{\frac{1}{2}} \mathrm{~cm}$.

An essay on journalism and freedom of the press versus corrupt government. Published by Chuchubi, Aruba, July 1960, 23 pp. $20 \times 13 \frac{1}{2} \mathrm{~cm}$. 
Antillen-nummer. Ons Zeewezen 5o, no. I, Jan. I96I, 84 pp. (abt. 36 pp. text) $27 \frac{1}{2} \times 20 \mathrm{~cm}, 45$ phot., col. cover excl.

Haven van Curaçao (p. 23-24, 26). Taak van de Koninklijke Marine (27, 29-30). Korps Mariniers en Nederlandse Antillen (31, 33, 35). Curaçao en de olie $(37,39,4 \mathrm{r})$. M.S. Antilia $(42-43,45)$. De K.L.M. in

"de West" (47). Enkele scheepvaartagenten op Curaçao (49-52).

Verlangen naar een sneeuwstorm (53-54). Havens van Aruba (55, 57-

59). Nederlandse Antillen als knooppunt van scheepvaartwegen (6I-

63). Scheepsbouw en industrie in de Nederlandse Antillen (65-67).

Appelman, H. \& Dirven, J. G. P. \& TJong A Hung, A. R.: Het Melkveeproefbedrijf op de Landsboerderij. Verslag $2 e$ halfjaar 1958. Versl. rapp. Dep. L.V.V. Sur. 42, 1960, iv + 12 + (19) pp. mimeogr.

Aruba Netherlands West Indies. Aruba Tourist Bureau, York Litho Miami, [folder] 12 pp. $20 \frac{1}{2} \times 23 \mathrm{~cm}$., col. ill.

Aruba, shopping fiesta, sunbeach siesta. Streetmap. Caribpress Advertising, Aruba Printshop, [folder] 24 pp. $21 \times 9 \frac{1}{2} \mathrm{~cm}$.

Aruba this week. A lodge publication. Edited by NANCy L. MAC EACHERN, Aruba Caribbean Hotel. Vol. I, No. I4, 22. IV. 196I, 6 pp. $30 \frac{1}{2} \times 22 \frac{1}{2} \mathrm{~cm}$.

Aruba tourist, commercial and industrial guide. Guia turistica comercial e industrial de Aruba. Year 2. No. 2, July 1960, ro,ooo copies. Daal's Pub. \& Trad Co.; Françors Citroen ed.; General Printing Co., Aruba (not for sale), 100 pp. $21 \times 15 \mathrm{~cm}$, ill.

Auble, Helen Lowe: Caribbean Vacationlands. Winter I96o-I96r, Volume roa. Caribbean Publications, St. Thomas, 130 pp. $21 \times 13 \frac{1}{2} \mathrm{~cm}$, ill.

Neth. Antilles p. 50-62, and p. II, 14 (col. fig.); Surinam p. 37-38.

BAshir, G. A.: Was Jezus God of mens? Surin. Islam. Vereen. Paramaribo, (1959), I6 pp. $19 \times 14 \mathrm{~cm}$.

Begroting van de Nederlandse Antillen voor het dienstjaar I96r. Staten van de Nederlandse Antillen. Zittingsjaar 1960-1961-17. Curaçao, 193 + 57 $+2+4$ pp. $25 \times 32 \frac{1}{2} \mathrm{~cm}$. Begroting ... 1962. Zittingsjaar 1961-196229. $195+47+2+4$ pp.

BEKENDAM, J.: Inductie van mutaties bij rijst door röntgenbestraling. (X-ray induced mutations in rice.) Diss. Wageningen I3. I. I96I, viii+ 68 pp., 14 graphs. - Also in Meded. Landbouwhogeschool Wageningen $6 r$.

BERG, H. VAN DEN: Tragedia di e boto "Gladys". Ig dia riba laman cu hamber, sed y solo abordo di "Gladys". Drukkerij Scherpenheuvel (Curaçao), (r96r), 39 pp. (incl. ro pp. adv.).

Biasutti, Renato: Le razze e $i$ popoli della terra, Vol. $I V$. Unione tipografico - Editrice Torinese, 1959.

America, p. 271-738, ill. - R. Biasurtr: I popoli degli istmi e delle Antille, p. 50I-522, fig. 378-390. - JosÊ ImBELLONI: Gli Amazzònici, p. $609-648$, fig. $455-487$, col. pl. $\mathrm{X}$ excl.

Billiton-Maatschappij I860-I960, see: KAMP.

Boekenweek Aruba $6 \mathrm{t} / \mathrm{m}$ ro juni 196r. Aruba Drukkerij N.V., 196r, 23 (32) pp., ill. 
Boekenweek Curaçao I96r. N. V. Paulus Drukkerij; Comité Boekenweek Curaçao 196r, Programmaboek Boekenweek Curaçao 196I, 26 mei - 3 juni. 24 (32) pp. $29 \times 22 \frac{1}{2} \mathrm{~cm}$, ill.

BOND, JAMEs: Birds of the West Indies. With colour illustrations by Don R. Eckelberry and line drawings by Earl L. Poole. Collins,

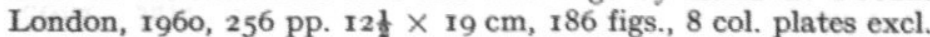

BRAASEM, W. A.: Sprookjes van Noord-en Zuid-Amerika. Ill. by H. DE Vos. C. P. J. van der Peet, Amsterdam, (1956), 208 pp.

Negro stories from Surinam (p. 125-139) and Curaçao (p. 175-182).

Brenneker, Pater V. Leketé minawá. Cantica dje dushi tempu bieuw. Boekhandel St. Augustinus, Curaçao.

23 Curaçao 'songs from the good old days'.

Brits Guyana en Frans Guyana. Landen documentatie ten dienste van het onderwijs in de aardrijkskunde, no. 29. Kon. Inst. v. d. Tropen, Amsterdam, 1960, 25 pp., fig.

Buisonjé, P. H. DE \& ZonNeveld, J. I. S.: De kustvormen van Curaçao, Aruba en Bonaire. (The coasts of Curaçao, Aruba and Bonaire.) Uitgaven Natuurwet. Werkgroep Ned. Antillen, No. II, 1960, 24 pp., ill. - From Nieuwe W. I. Gids 40.

Bulletin No. 74. Landbouwproefstation in Suriname, April 1957, 210 pp. ill., folding maps. VAN DER VOORDE, Soil conditions..., diss. Wageningen. Bulletin 77, June 1961, (ro) pp., 2 pls. excl. van Hoof, from T. Plantenziekten 65; DiRven \& VAN HOOF, from T. Plant. 66.

Bulletin 78, May 196r, (13) pp., 3 figs. van Amson, from $S L$.

Bunitesa den secura. Schoonheid van een droog klimaat. (Drought's beauty.) Willemstad, maart 196r, (28) pp. I6 $\frac{1}{2} \times 25 \mathrm{~cm}$, ro phot.

Guide to Brother M. ARNOLDo's exhibition of photographs.

Butner, C.: Goud in het Slangenbos. 'Oerwoud Serie' Van Goor Zonen, Den Haag, $5_{2}$ pp., ill. by TILly Dalton, map. [Boy's book.]

Centrale Bank van Suriname. Verslag over 1957, Paramaribo, (1958), I 19 pp., $24 \times 16 \mathrm{~cm}$, folding table excl. [Ist report] - 1958, (I959), I47 Pp. - I959, (1960), I53 pp. - I960 (I961), I57 pp.

Citroen, F., see: Aruba tourist guide.

Coomans, H. E.: Jaarverslag 1959 Stichting Wetenschappelijke Bibliotheek. Willemstad, 1960, 19 pp. $21 \times 14 \mathrm{~cm}$ mimeogr.

Culturele Kroniek. Cultureel Centrum Curaçao, Curom, Curaçaosche Kunstkring, Filmliga Curaçao, Wetenschappelijke Bibliotheek. Printed by Cur. Courant N.V., Curaçao. Eerste Jaargang no. I, May I96I, 32 pp. $29 \times 21 \mathrm{~cm}$, cover excl., ill. -2 , June -3 , July.

Current Caribbean Bibliography 8, 1958. Car. Comm., Puerto Rico, 196r, viii +90 pp. $23 \frac{1}{2} \times 17 \mathrm{~cm}$. An annual review of bibliography. Cumulative issue 1954-1958. - IRMA HANNAYs ed.

De Bonte Vlinder I96r. [Calendar] Paramaribo, (1960), I + 12 pp. $32 \times$ $22 \mathrm{~cm}$. 
Debrot, Cola: Mijn zuster de negerin. Literaire pocketserie no 63 , Uitgeverij De Bezige Bij, Amsterdam, r96r, 85 pp. $18 \times$ ro $\frac{1}{2} \mathrm{~cm}$.

De Curaçaosche Bank. Verslag over het jaar 1959. (Curaçao, 1960), 59 pp., 2 folding tables excl.

De Heilige Profeet Mohammad s.a.w. (korte levens beschrijving). Surin. Islam. Vereen. Paramaribo (1956), 57 pp. I8 $\times$ I4 cm., fig.

De Islamietische vrouw en haar recht. Surin. Islam. Vereen. Paramaribo, (1957), 20 pp. $20 \times \mathrm{I}_{4} \mathrm{~cm}$, fig.

De Nederlandse Antillen en Nederland. Economische Voorlichting No. 9, 3. III. 196r, $4^{8}$ pp. incl. abt. 22 pp. adv.

De Nickeriaan, Orgaan van de Nationale Vereniging "Nickerie Vooruit." Jubileum nummer, 20e Jaargang, 4. VI. r96r, 30 pp. $34 \times 22 \mathrm{~cm}$ mimeogr. 4 pp. with 7 phot. excl.

De rechtsorde in het Koninkrijk der Nederlanden (de basisregelingen). Second rev. edition of Schakels $S_{3}$ I/NA 26. Schakels $S_{39 / N A} 32$, 1960, 192 pp. $22 \times 16 \mathrm{~cm}$.

De Slaventijd in Odo's. Een boekje over Suriname's heden en verleden. Samengesteld door het Bureau Volkslectuur. 'Creoolse Wijsheid' I. Radhakishun \& Co, Paramaribo 196o, (ii) + r7 + (i) pp., ill.

De Surinaamsche Bank N.V. Aanvullend Verslag over het boekjaar 1955, (Paramaribo), 26 pp. $23 \frac{1}{2} \times 15 \frac{1}{2} \mathrm{~cm}$. - Verslag 1956, (1957), 45 pp., folding table excl.

De Vlag van de Nederlandse Antillen. The Flag of the Netherlands Antilles. La Bandera ... Bandera.... Regerings Voorlichtingsdienst Ned. Ant. (Curaçao), [folder] 6 pp. $22 \frac{1}{2} \times 18 \frac{1}{2} \mathrm{~cm}$, col. fig.

Diekmann, Miep: Anders is niet altijd beter. Roman voor oudere meisjes. U.-M. "West-Friesland", Hoorn, (1954), 2 II pp. Drawings by EmILE BRumsteEdE. [Girls'book, dealing with Curaçao.]

Diekmann, Miep: The Haunted Island. London, Methuen \& Co. Ltd. 1959. Translation of "De Boten van Brakkeput" by A. J. Pomerans, II9 pp.; ill. by JENNY DALENOORD. [Children's book, dealing with Curaçao.]

DONNER, WALTHER ROËL WILlIAM: The financial mechanism of the Netherlands Antilles. Diss. Amsterdam 23. VI. I96I, Uitgeverij Werto, Amsterdam, 196 pp. $30 \times 21 \mathrm{~cm}$ photopr.

Droogleever Fortuyn, A. B.: Age, stature and weight in Surinam conscripts. Meded. Kon. Inst. v. d. Tropen ror, Afd. Cult. Phys. Anthrop. $44,1952,126$ pp. $22 \times 15 \frac{1}{2} \mathrm{~cm}$ photopr.

DU Ry, C. J. \& VAN HEEKEREN, H. R.: Studies on the archaeology of the Netherlands Antilles. I \& II. Uitgaven Natuurwet. Werkgroep Ned. Antillen, No. 1o, 1960, 40 pp., ill. - From Nieuwe W. I. Gids 40.

Economic review Hollandsche Bank-Unie N.V., Neth. Antilles., $27 \frac{1}{2} \times$ $21 \mathrm{~cm}$ mimeogr. I27-128, 1959, $5+6$ pp.; 129-130, 1960, $6+6$ pp.; $I_{3}-132, \mathrm{r961}, 6+6 \mathrm{pp}$. 
Economische Voorlichting 55, no. 9, 1961, see: De Nederlandse Antillen en Nederland.

Economische Voorlichting Suriname. Uitgave van het Departement van

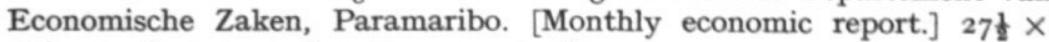
$2 \mathrm{I} \frac{1}{2} \mathrm{~cm}$ mimeogr. $I$, Jan. 1954 , no. $I$, 18 pp.; no. 2 , I5 pp.; no. 3, I9 pp.; no. 4, 17 pp.; no. 5, 24 pp.; no. 6, 20 pp., etc.; no. 12 , Dec., 25 pp. -2 , Feb. 1955, no. 3, 27 pp; no. 4, 24 pp., etc.; no. 7, 30 pp.; no. 8, 29 pp.; no. 9, 35 pp. $-3,1956$, 12 nrs., abt. 25-30 pp. each; 4, 1957, 12 nrs, 24-36 pp. each; Extra-nummer, 25 pp. -5 , 1958, I2 nrs., 24-31 pp. each. $-29 \frac{1}{2} \times 20 \frac{1}{2} \mathrm{~cm}$, printed. 6, no. I, jan. 1959, 23 pp. excl. cover; no. 2, 15 pp., 15-23 pp. each. -7 , 1906, no. $1-7$, 18-20 pp. each, I2 nrs.

Elmar 1950-1960. N.V. Electriciteits-Maatschappij 'Aruba'. Aangeboden aan onze verbruikers ter gelegenheid van het ro-jarig bestaan. $30+30 \mathrm{pp}$. $30 \times 21 \frac{1}{2} \mathrm{~cm}$, ill. [Dutch - Papiamento.]

Evans, CllfFord \& Meggers, Betty J. : Archeological investigations in British Guiana. Smiths. Inst. Bureau of American Ethnology, Bulletin 177, Washington 1960, xviii + 418 pp., 127 figs., 68 pls. excl.

Faverus, H.: Persoonlijkheidsvormen in het Caraibisch gebied en Afrika gezien in het licht der Westernisatie. ABUWA, Sociaal-Economische Werkgemeenschap, Amsterdam, 1960, 33 pp.

Flornoy, Bertrand: Jivaro. Onder de koppensnellers van de Amazone. Scheltens \& Giltay, Amsterdam, (1954), 223 pp., figs., 16 phot. on ro pls. excl. Translated by J. F. KLIPHUIS from Haut-Amazone, Plon, Paris, 1939, 273 pp., 12 pls.

Plate opposite p. 17 of translation showing a Surinam Indian!

Fly Caribair to St. Maarten. Caribair, New York, 1960, [folder] 6 pp. col. litho $15 \frac{1}{2} \times 9 \mathrm{~cm}$.

Fodor, Eugene (ed.): Fodor's Guide to the Caribbean, Bahamas and Bermuda. I96r. Fodor's Modern Guides, Inc., Printed in the Netherlands - Mouton \& Co., The Hague, xvi + lxvi + p. 39-657, vignets, 25 maps \& 12 city plans, many plates in colour and black and white excl., large folding map.

Surinam, p. 548-557 (by Albert Helman); Dutch West Indies, p. 592-623; plates facing p. 54I (col.), 612.

Foto-album van Curaçao I837-1962, see: HARTOG, Het verhaal der Maduro's.

Franke, S.: Anansie. De avonturen van Heer Spin in Suriname. H. ten Brink's Uitgeversmaatschappij, Meppel, $5_{5}^{8}$ pp. [Children's book.]

Freitas, C. M. DE: De Antilliaanse studerende jeugd in Nederland. (Sittard, 196r), 36 pp. $27 \times 21 \mathrm{~cm}$ mimeogr.

Gedenkboek Koninklijke Nederlandse Vereniging "Onze Vloot". Afdeling Curaçao 1907-1957. 71 pp. $24 \times 15 \mathrm{~cm}, 51$ figs.

Gedichten bekroond door het Prins Bernhard Fonds Suriname. Uitgegeven ter gelegenheid van de 5 oste verjaardag van Z.K.H. Prins Bernhard. (Paramaribo, 196r), Drukkerij Eldorado, 7 pp. 
Poetry by M. A. Slory, A. E. Gilds, W. Grimmèr, E. C. Husband, and J. E. Pont in Surinam Vernacular.

Geijskes, D. C.: De expeditie naar de Tafelberg in 1958. Schakels $S 45$, Sept. I96r, p. 2-13, 4 phot.

Gids voor Moeslim-jeugd. Een korte samenvatting der Islamitische grondbeginselen. Vierde druk. Surin. Islam. Vereen. Paramaribo, (1955), 20 pp. $18 \times 12 \mathrm{~cm}$, fig.

Goslinga, C. Ch. \& Noort, A. C. H. J. van \& Sjak Shie, H. E.: Geschiedenis van Amerika I voor de middelbare school. J. B. Wolters, Groningen, 196r, I59 pp.

GrAAF, H. J. DE (ed.): Nederlanders over de zeeën. 350 Jaargeschiedenis van Nederland buitengaats. 2nd edn. W. de Haan N.V., Utrecht, I955, 260 pp. ill.

W. R. Menkman: Nederland in Amerika en West Afrika, p. 9-8I, 9 pls. excl.

Ha-ki-ka-toes-swalaat. Surin. Islam. Vereen. Paramaribo (1954), 20 pp. $2 \mathrm{I} \frac{1}{2} \times 13 \frac{1}{2} \mathrm{~cm}$.

Hamburgisches Welt-Wirtschafts-Archiv. Auslandskunde. Literaturnachweis über die Gebiete Wirtschaft und Politik, Recht und Technik. LänderAbteilung No. 12. Karibische Inseln T. 2: Europäischer und U.S.A.-Besitz. I. Hälfte: Das Gebiet im ganzen und Britischer Besitz; vi $+\mathrm{I}_{3}$ pp. 2. Hälfte: Französischer und Niederländischer Besitz, Puerto Rico und Amerik. Jungfern-Inseln; v + I3I pp. $29 \times 20 \frac{1}{2} \mathrm{~cm}$ mimeogr.

Niederländisch-West-Indien im Ganzen und Niederländische Antillen, ii + p. 27-48. Surinam (Niederländisch-Guyana), ii + p. 49-7I.

HARTOG, JoH.: Het verhaal der Maduro's en Foto-album van Curaçao I837r962 uitgegeven ter gelegenheid van het honderdvijfentwintigjarig bestaan van S.E.L. Maduro \& Sons N.V. en opgedragen aan de Bevolking van de Nederlandse Antillen. The Story of the Maduros and Photo Album of Curaçao 1937-1962 published on the occasion of the hundred and twenty-fifth anniversary of S.E.L. Maduro \& Sons, Inc. D. J. de Wit, Aruba, (Jan.) $1962,179+(60)$ pp. $26 \frac{1}{2} \times 19 \frac{1}{2} \mathrm{~cm}$, ill.

HeinsdijK, D. \& Hulster, I. A. DE \& SniJders, W. J. J.: De inschakeling van het bos voor de economische opbouw van Suriname. Nota... Paramaribo, Aug. 1950, $23+(19)$ pp. mimeogr. $35 \times 22 \mathrm{~cm}, 2$ folding handcol. maps. - Note on Activating the forest for the economical development of Surinam. 5 pp. added.

Helman, Albert: Caraibisch passiespel, in vijf acten met een proloog en epiloog. N.V. Varekamp \& Co. Paramaribo, 1960, 98 pp. $17 \times 11 \frac{1}{2} \mathrm{~cm}$, I5 plates excl.

Het Islamietisch Geloof. Surin. Islam. Vereen. Paramaribo, (1958), 36 pp. $19 \frac{1}{2} \times 14 \frac{1}{2} \mathrm{~cm}$.

Het verhaal der Maduro's, see: HARTOG.

Heyligers, Petrus Cornelis: Vegetation and soil of a white sand savanna in Suriname. Diss. Utrecht 2. X. 1961, vi $+(79)+(9)$ pp., $34 \times 21 \frac{1}{2} \mathrm{~cm}$ 
mimeogr., 5 loosely inserted tables \& 31 separate maps in blue print, 27 phot.

To be published as vol. III, part I of 'The Vegetation of Suriname'.

Hilgers, Johan Herman Christiann: Pterygium on the island of Aruba. Diss. Amsterdam 9. VII. I959, I44 pp., drawings, I4 figs., 8 col. phot. excl.

Hollandsche Bank-Unie N.V. Jaarverslag I959-1960, 46, Amsterdam, (Nov. 1960), 219 pp. $30 \times 23 \mathrm{~cm}$, ill. - Jaarverslag I960-I96I, 47, I96I, I80 pp.

Holland Bank Union. - Neth. Ant. 46 p. $64-77,2$ phot.; 47 p. $68-79$,

2 phot. Surinam 46 p. 78-95, 2 phot.; 47 p. 80-91, 2 phot.

Hooghiemstra, E.: Trainingscursus mond- en klauwzeer, Columbia, juni 1960. Versl. rapp. Dep. L.V.V. Sur. 48, 196r, 43 pp. mimeogr.

Hotel and Casino Aruba Caribbean. Harris \& Company Adv. Inc. Col. [folder] 12 pp. $23 \times 10 \mathrm{~cm}$.

Hulster, I. A. DE: Jaarverslag 1959 Dienst Landsbosbeheer Suriname. (Annual report 1959 Forest Service Suriname.) (Paramaribo, 1960), $4^{8}+\mathrm{xi}$ (summary) $+(26)$ pp. $27 \times 21 \mathrm{~cm}$ mimeogr., 55 figs. on 32 pls. excl., cover picture. - Jaarverslag I96o (Annual report 1960) Paramaribo, I96I, ii + 6I + xiii (summary) + (19) pp. mimeogr., 72 figs. on 46 pls. excl., cover picture.

Hungerford, H. B.: The Corixidae of the Western Hemisphere (Hemiptera). University of Kansas Science Bulletin 32, 1948, 827 pp., III pl. incl.

Also dealing with material from Surinam and Antilles.

Husson, A. M.: De zoogdieren van de Nederlandse Antillen. Mammals of the Netherlands Antilles. Uitgaven Natuurwet. Werkgroep Ned. Antillen, No. I2, Dec. 196o. Nijhoff, 's-Gravenhage; Boekhandel Salas, Curaçao; viii +172 pp. $19 \frac{1}{2} \times 13 \mathrm{~cm}, 27$ figs., 42 pls. \& 4 maps excl.

Ibero-Amerikadag. Utrecht I9-X-I96I. (Spaans, Portugees en IberoAmerikaans Instituut der Rijksuniversiteit te Utrecht), 2 vols., $13+3^{8}$ pp. $20 \frac{1}{2} \times 14 \frac{1}{2} \mathrm{~cm}$ photopr.

Discurso de S.A.R. el Principe de Los Países Bajos, p. I-5; Discurso del Excmo. Sr. Dr. H. R. van Houten, p. ro-rir. - Dr. Silvio Zavala, El Nuevo Mundo en los intercambios mundiales postcolombinos, p. I-I3; Z. Exc. Dr. Carlos Cyrillo Junior, Brasilia, p. 27-32. - With translations in Dutch.

Investeringsverordening voor Suriname. Met bijbehorende toelichting. I96o. Vereniging Surinaams Bedrijfsleven, Paramaribo, 196o, 28 pp.

Jaarverslag Dienst Landsbosbeheer, see: DE HULSTER.

Jaarverslag 1959 Tienjarenplan Suriname. Stichting Planbureau Suriname, Paramaribo, Dec. 1960, r9o pp., ill., folding tables.

JAPING, C. H. \& JAPING, H. W.: Houthandboek Surinaamse houtsoorten. Dienst 's Lands Bosbeheer Suriname, Paramaribo; printed by H. Veenman en Zonen N.V., Wageningen, I960, 264 pp. $24 \frac{1}{2} \times 17 \mathrm{~cm}, 176$ figs., frontispice. 
JESSURUN, FRITS: Beschouwingen over de aetiologie van psychiatrische syndromen bij stoornissen van de temporale hersenen. (Aetiology of psychyatric syndromes in disturbances of the temporal lobe, including the rhinencephalon.) Diss. Groningen 28. VI. I961, xii +312 pp., 8 figs.

The author is of Surinam birth and got his first medical education at Paramaribo.

Juliana, Elis: Aventura di un Kriki. Contá pa e mes. Curaçao, September 1960, 46 pp. $20 \frac{1}{2} \times 14 \mathrm{~cm}$, cover excl.

Jul[I]ANa, Elis: Dama di Anochi. I7 suspiro den kietud di anochi especial pa damas. Editá pa: S.V. Van Engelen, Curaçao, 1959, I9 pp. $20 \frac{1}{2} \times$ $14 \frac{1}{2} \mathrm{~cm}$, cover excl. [Papiamentu poetry.]

Juliana, Elis: Flor di Anglo. Poësia y sketch. Drukkerij Scherpenheuvel, Curaçao, I96I, 80 pp. $21 \times$ I4 cm. excl. cover. [Papiamentu poetry.]

Juliana, Elis: Maka taka. Editá pa G. C. T. van Dorp \& Co, (Ned. Ant.) N.V., Curaçao, I96I, III pp. $19 \times 12 \mathrm{~cm}$.

Kamer van Koophandel en Fabrieken, Paramaribo, Suriname. Jaarverslag 1955, (1956), $5^{2}$ pp. $23 \frac{1}{2} \times 16 \mathrm{~cm}$. - Jaarverslag 1956, (1957), 72 pp., folding tables excl.

KAMP, A. F.: De standvastige tinnen soldaat. $1860-1960$ / N.V. Billiton Maatschappij 's-Gravenhage. 297 pp. $28 \times 22 \mathrm{~cm}$, ill., I4I phot. excl. N.V. Billiton Maatschappij Suriname, p. 227-245, map \& phot. 7 I-Io6 on 34 pp. excl.

Keuls, Hans: Plantage Tamarinde. Toneelspel in drie bedrijuen. Bond van Nederlandse Toneeluitgevers, 1957, 88 pp. $19 \frac{1}{2} \times 13 \frac{1}{2} \mathrm{~cm}$.

Keur, John Y. \& Keur, Dorothy L.: Windward Children. A study in human ecology of the three Dutch Windward Islands in the Caribbean. Van Gorcum Ltd., Assen, 1960, viii + 299 pp., 4 maps, one of which excl., I I pl. excl. - With a foreword by VERA RUBIN, p. v-viii.

Klinkhamer, K.: De Caraïbische Spraakkunst. Amsterdam, dd. 25. III. 1955, (4) +53 pp. $33 \times 20 \mathrm{~cm}$ mimeogr.

Koninklijk Instituut voor de Tropen, Amsterdam. Negen en veertigste Jaarverslag I959. Amsterdam (1960), 78 pp., 5 pls. excl. - Vijftigste Jaarverslag 1960, (1961), 84 pp., 7 pls. excl.

KRUIJER, G. J.: Suriname en zijn buurlanden. Lichtplekken in het oerwoud van Guyana. 3rd. edn. J. A. Boon \& Zoon, Meppel, r96o, 286 pp., figs., 23 phot. on 15 pls. excl. - Ist. edn. I95I, 285 pp.; 2nd. edn. I953, 292 pp., 23 phot.

KuIL, H.: De bestrijding van teken bij rundvee. Versl. rapp. Dep. L.V.V. Sur. 45, Dec. 1960, $23+$ (II) pp., fig., mimeogr.

KuIL, H.: De kunstmatige inseminatie bij het rund, I952 tot I959. Versl. rapp. Min. L.V.V. Sur. 52, 1961, $20+(32)$ pp. mimeogr.

KuIL, H.: Onderzoek naar het voorkomen van bacteriële uierontstekingen bij melkvee. Versl. rapp. Min. L.V.V. Sur. 53, 1961, 23 pp. mimeogr.

Lago Telephone Directory, Lago Oil \& Transport Company, Ltd., Aruba, I96I, iv $+55+4$ pp. $2 \mathrm{I} \times 13 \frac{1}{2} \mathrm{~cm}$. 
LAKERVELD, W. L. VAN: Enkele aspecten van de industrialisatie van Suriname. (Planbureau Suriname) 's-Gravenhage, 1959, 66 pp. mimeogr. LAMPE, Padú: The 3rd Element. A Reflection Book; Carlton Press, New York, 1960, xvi +79 pp. $20 \times 13 \frac{1}{2} \mathrm{~cm}, 4$ figs., dust cover ill.

"A revolutionary hypothesis propounding the existence of a supernormal form of vibration, serving as a link between and interpenetrating the two worlds, while playing a fundamental role in all processes and manifestations in nature" - by Aruba's 'Padú del Caribe': composer-pianist, and painter.

Landbouwproefstation, Suriname. Jaarverslag 1959. Meded. 22, 1960, II6 pp., figs., I2 pls. excl.

van Emden, ed.; van Amson, Appelman, Dirven, van Doesburg, Ehrencron, van Hoof, ter Horst, Schouten, Small, van SuchteLEN, and UBELS,

Landbouwproefstation, Suriname. Jaarverslag I960. Meded. 25, 1961, 92 pp., II pls. excl.

van Emden, ed.; van Amson, Dirven, van Doesburg, van Dijk, Ehrencron, van Hoof, ter Horst, Huiswoud, Mastenbroek, del Prado, Schouten, Small, van Suchtelen, and Ubels.

Landsverordening. ... kiesrecht en de verkiezing van de leden van de eilandsraden Bonaire en de Bovenwindse Eilanden. Publicatieblad 1959, 20, 59 pp. - Landsbesluit... Kiesreglement Eilandsraden Bonaire en Bovenwindse Eilanden. P.B. 1959, 21, (72) pp.

Lauffer, Pierre: Njapa. Imprenta di Skèrpènè, (Curaçao), r96r, 79 pp. $21 \times 14 \frac{1}{2} \mathrm{~cm}$.

30 short stories, 6 poems.

LAuffer, Pierre: Wiri-wiri. Imprenta di Skèrpènè, (Curaçao), 196I, 64 pp. $21 \times 14 \mathrm{~cm}$.

27 short stories, 5 poems.

Lawaetz, Erik \& Lawaetz, Jenny: St. Martin, Caribbean island gem. Photographs by Fritz Henle. Printed in U.S.A., 20 pp. incl. cover $20 \frac{1}{2} \times 25 \frac{1}{2} \mathrm{~cm}$, 19 phot., 3 of them col.

LEGÊNE, J. J.: Herdenkingsnummer 225 jaar Suriname-Zending. (Zeister Zendingsgenootschap, Zeist), r96o, 12 pp. ill., map excl.

LE PAGE, R. B.: Jamaican Creole. An historical introduction to Jamaican Creole, and four Jamaican Creole texts by DAvid DE CAMP. Creole Language Studies I, London, MacMillan \& Co Ltd, I96o, x + I82 pp.

Curaçao and Surinam only shortly mentioned.

Lijst van lopende tijdschriften en series handelende over Latijns Amerika en aanwezig in de Centrale Boekerij van het Koninklijk Instituut voor de Tropen Amsterdam, 1960, 46 pp. mimeogr.

Lionarons, H. : De visserij in Brits Guyana, in het bijzonder de productie, de opslag en de afzet van vis in Georgetown. (Versl. rapp.) Min. L.V.V. (Sur.) 54, 196r, 17 pp. mimeogr., fig.

LUYKEN, R.: Nutriënt-behoefte van enkele Surinaamse bevolkingsgroepen. Centraal Instituut voor Voedingsonderzoek T.N.O., Utrecht, Rapport R I2I7, I4 Pp. mimeogr. 
L.V.V. in 1958, and L.V.V. in 1959, see: DEL PRAdo.

Maduro, Antorne J.: Loque a sobra den e Macutu di dicho, refran, proverbio, frase $i$ palabranan di nos lenga $i$ nan nificacion na ulandes. Editor: Ciro J. Cardoze, Corsou 1960, 212 pp.

Maduro, Antorne J.: Spaanse Documenten uit de jaren I639 en I640 betrekking hebbende op de voorgenomen herovering van het eiland Curafao door de Spanjaarden. Documentos de los años 1639 y r64o que se encuentran en el Archivo General de Indias en Sevilla, referentes a los designios que tenían los españoles para la reconquista de la isla de Curazao, conquistada por los holandeses el 29 de julio de r634. Niet in de handel. Oplaag 400 ex. I96r, Drukkerij Scherpenheuvel, Curaçao, II4 pp. $24 \times$ $15 \frac{1}{2} \mathrm{~cm}$, map.

Mancius, W. van (W. van Mannekus): Olie op de golven. N.V. Arbeiderspers, Amsterdam, 196r, 267 pp.

A novel, also dealing with Curaçao.

Maria Sibilla Merian-kalender Ig6o. N.V. Levensverzekering Maatschappij 'Utrecht', (1959), [4-leafed calendar] $24 \times 16 \mathrm{~cm}, 4$ col. repr., 4 pp. text.

From Merian: Over de voortteeling en wonderbaerlijke veranderingen der Surinaamsche insecten, 1647-1717, pls. 9, 15, 31, and 60.

MARTINUS FRANK: Ta amor so por. Un korona di poesia traha riba un isla ku no ta eksisti meimei di Hulanda ku Korsow. Boekhandel Salas, Curaçao, March 196r, 32 pp. $21 \times 13 \mathrm{~cm}$, excl. cover. [Papiamentu poetry.]

Marugg, TIP: Weekend Pilgrimage. Translated from the Dutch by Roy EDwards. Hutchinson \& Co, London, 1960, I9I pp. $19 \frac{1}{2} \times 12 \frac{1}{2} \mathrm{~cm}$.

Maulana Mohammad Ali: Mirza Ghulam Ahmed van Qadian. Zijn leven en zijn missie. Vertaald uit het Engels door Twahir Mohammed AJoeb. Sur. Islam. Vereen. Paramaribo (1958), iv + 34 pp., 2 figs.

Mededeling No. 22. Landbouwproefstation Suriname, June r96o, Ir6 pp., ill. Jaarverslag 1959.

Mededeling 23, July 1961, 44 pp., ill. van Amson, Bodemonderzoek... Cacao.

Mededeling 24, Aug. 1961, 73 pp., ill. Samson, Handleiding ... citruscultuur...

Mededeling 25, Aug. 196r, 92 pp., ill. Jaarverslag 1960.

Mededeling 26, Oct. I96I, 59 pp., ill. UBELs, Modernisering... rijstbouw in Nickerie.

Mededeling 28, Dec. 1961, 57 pp., ill. Small, Bacoven studiereis.

Meijer, J.: M. J. Lewenstein's Opperrabbinaat te Paramaribo (I857/81864). Analyse van het Surinaamse Jodendom in zijn crisisperiode. Uitgeverij De Driehoek, Amsterdam, (r959), r38 pp.

MIRvish, RoBert F.: Hotel Internacional. (Roman). Nederlandsche Keurboekerij N.V., Amsterdam, 1953, 270 pp. [Novel.] - Translation of $A$ house of her own, by JAN DE WINTER. Dealing with Curaçao, p. 103-2ro.

Mohamad Yabub Khan: De gouden daden van de Islam. (vertaald uit het Engels). Surin. Islam. Vereen. Paramaribo (1959), $3^{8}$ pp. $\mathbf{1}_{7} \frac{1}{2} \times \mathbf{I}_{4} \mathrm{~cm}$. 
Monthly Bulletin. Ionospheric, geomagnetic, earth current, solar radio noise, solar activity data. Paramaribo, Surinam. Printed at the Royal Netherlands Meteorological Institute, De Bilt. April $195^{8}$ (no.ro), vi + 28 pp.; May, vii +25 pp.; July, ix +27 pp.; August, xiii +30 pp., all publ. 1960. - September 1958, viii +28 pp.; October, xi +29 pp.; November, $\mathbf{x}+27$ pp.; December, ix +29 pp., all publ. 1961. - January 1959, iv +28 pp.; February, iv +27 pp., all publ. r96r.

Monthly publication for Trade and Industry of Curafao. Maandblad voor de Handel en Nijverheid van Curaçao. Curaçao Association of Trade and Industry. Augustus 196r, r6 pp. excl. cover, ill. - Juli [last number with Dutch title and without special cover.]

Nederland en de Tropen. Tropenmuseum Amsterdam I96o. 50 jaar Koninklijk Instituut voor de Tropen. 24 pp. $23 \times 13 \mathrm{~cm}$.

Nederlandsche-Handel-Maatschappij N.V., Verslag over het boekjaar 1959. Amsterdam, 1960, 84 pp. $29 \frac{1}{2} \times 23 \mathrm{~cm}$, ill.

Surinam, p. 32-35; Neth. Antilles, p. 36-39.

NIET, M. DE: Alfabetisch Register op het Gouvernementsblad van Suriname. Jaargangen $1816-1958$. Staatsdrukkerij en Uitgeversbedrijf, 's-Gravenhage 1959, xvi + 47I pp. - Aanvullingen Jaargang 1958 , II pp.

Nijenhuis, Lourens Evert: Blood group frequencies in the Netherlands, Curaçao, Surinam and New Guinea. A study in population genetics. Diss. Amsterdam 13. XII. 1961, 203 pp., 28 sketch maps.

Curaçao and Surinam: p. 53-76, 126-127, 165-173, 201.

Nos Isla. Personeelsblad van Shell Curaçao N.V., etc. Printed by 'De Curaçaosche Courant' N.V., Curaçao, $38 \times 27 \frac{1}{2} \mathrm{~cm}$, ill. [New bi-weekly.] Ist year, no. $I$, I4. I. I96I, 6 pp.; 2, 28. I. -22 , I8. XI., 4 pp. each.

Observador. Semanario informativo netamente Arubiano. Editá y publicá door di: Publicidad "Exito" Aruba A. H., 22. XI. I96I, I6 pp. $29 \frac{1}{2} \times 22$ $\mathrm{cm}$, ill., cover excl.

Oduber, Frederico: Beseffend. Uitgave D. J. de Wit, Oranjestad, Aruba, (196r), 28 pp. $21 \frac{1}{2} \times 12 \mathrm{~cm}$. [Poetry.]

OFFERHAUS, H.: De realisering van de taakuitbreiding van het Koninklijk Instituut voor de Tropen. (Kon. Inst. Tropen) Amsterdam, 1961, I I pp. $22 \times 14 \frac{1}{2} \mathrm{~cm}$.

Ogrizer, Doré: L'Amérique du Sud. Tome I. Brésil Venezuela Colombie Equateur Guyanés. Le Monde en couleurs. Odé, Paris, 1957, ill.

La Guyane Hollandaise, p. 389-39r.

Ondernemingslandbouw in Suriname. Dept. L.V.V.; Ver. Sur. Bedrijfsleven, Paramaribo, 1960, 28 pp., 13 figs., col. folding map.

Ons Zeewezen, see: Antillen-nummer.

Onze Vloot, Afdeling Curaçao, see: Gedenkboek r9o7-1957.

Opgave van litteratuur betreffende Suriname; 59 pp. - Opgave van litteratuur betreffende de Nederlandse Antillen; 35 pp. - Sticusa, Amsterdam, I. VII. I96I, $27 \times 21 \mathrm{~cm}$ mimeogr. 
Overeenkomst inzake een programma van samenwerking voor economische ontwikkeling tussen de Landsregering van Suriname en de Foreign Operations Administration van de Verenigde Staten van Amerika, 1954, Paramaribo, (8) pp.

Panday, Radjnarain Mohanpersad Nannan: Agriculture in Surinam 1650-1950 (An inquiry into the causes of its decline). Diss. Amsterdam 3. VII. I959, H. J. Paris, Amsterdam, xii +226 pp.

Petzoldt, T. R. \& Samson, H. A.: Suriname en het Statuut. Bestemd voor de Lagere Scholen. Dienst V. en V. Paramaribo, Dec. I954, I6 pp. $21 \frac{1}{2}$ $\times \mathrm{I}_{3} \frac{1}{2} \mathrm{~cm}$.

Petzoldt, T. R. \& SAmson, H. A.: Suriname en het Statuut. Bestemd voor de Middelbare Scholen. Dienst V. en V. Paramaribo, Dec. 1954, 68 pp. $2 \mathrm{I} \frac{1}{2} \times 13 \frac{1}{2} \mathrm{~cm}$.

Pine, Wilfred H.: An examination of farm management, marketing of agricultural products, and related problems in Surinam. Surinam American rechnical Cooperative Service, Paramaribo, 1960, 38 pp. mimeogr. (Rapport, Min. L.V.V.)

PRAdo, H. Del: L.V.V. in 1958. Sur. Landb. 8, 2/3, 1960, p. 25-93 + 39 pp., 3I figs.

Prado, H. Del: L.V.V. in 1959. Sur. Landb. 8, 6, 1961, p. 217-300 + 36 pp., $4^{8}$ figs.

PRAdo, H. DEL: Verslag van de Third Meeting on Cooperatives in the Caribbean... Versl. rapp. Dep. L.V.V. Sur. 44, 1960, i + 19 pp. mimeogr.

Puissesseau, RenÉ: Quelqu'un mourra ce soir aux Caraĩbes. Gallimard, Paris, 1957, 245 pp. - Someone will die tonight in the Caribbean. Translated from the French by STEPHEN Becker. Alfred A. Knopf, New York, 1958, 212 pp.; W. H. Allen, London, 1959, I90 pp.

Saba mentioned.

Radio Kelkboom. Año 7, Maart 1961, No. 3, (Aruba) 32 pp. $28 \times 21 \frac{1}{2} \mathrm{~cm}$, excl. cover, ill.

Ranis, J.: De Curaçaose jeugd en haar vrije tijdsbesteding. Sittard, r96o, 29 pp. $29 \times 21 \mathrm{~cm}$ mimeogr.

Rapport betreffende de malariaproblematiek in verband met het Brokopondoproject, see: VAN THIEL.

Rapport der Commissie... ter uitvoering van Motie I, aangenomen tijdens de derde openbare vergadering der Conferentie Nederland-Suriname Curaçao. (Commissie Aruba-Curaçao.) Curaçao, 1948, (2I) pp. $29 \times 2$ I $\mathrm{cm}$.

Records of forest plantation growth in Mexico, the West Indies, and Central and South America. Caribbean Forester, vol. 21 Suppl., Dec. 1960.

Not dealing with Surinam and Neth. Antilles.

Roethof, H. J.: Suriname bouwt aan zijn toekomst. Schakels $S 42$, 196r, 28 pp., ro figs., cover fig. excl. 
Rolle, F. J.: Status of zoological research in the Caribbean. Institute of Caribbean Studies, University of Puerto Rico, Mayagüez, P. R.; $28 \times 21 \frac{1}{d}$ cm mimeogr. No. $\boldsymbol{I}$ - January I96r, 9 pp. ; 2 - June, 17 pp.

Rosenstand, E. E.: Cuentanan Rubiano. General Printing Co. (Aruba), (I96r), 93 pp. $21 \times 15 \mathrm{~cm}$.

A number of Aruban children's stories in Papiamentu, broadcasted via Radio Voz di Aruba in the 'Ora Infantil'.

Rostra Economica, Maandblad Studiever. Econ. Fac. Univ. Amsterdam, Suriname nummer. Ioe Jaargang Nr. 39, Juli I96I, 36 pp., ill.

Articles by De Wit, Tiggelman, Meijer, Douglas, Speckmann.

Roth, Vincent: Tales of the trails. Georgetown, 1960, vi + r65 pp. $2 I \times 13 \frac{1}{2} \mathrm{~cm}$, frontispice, folding map.

Pages extracted from author's personal journal for 1918-192I, including some observations that may prove of interest of zoologists, ethnologists and sociologists.

SAher, Lilla van: Macamba. Saturn Press, London, 1950, 264 pp. 20 $\times \mathbf{r} 3 \frac{1}{2} \mathrm{~cm}$.

Samson, J. A.: Handleiding voor de Citruscultuur in Suriname. Meded. Landbouwproefsta. Sur. 24, 196r, 76 pp., r9 figs.

Samson, J. A.: Nederlandse en Amerikaanse impressies. Versl. rapp. Dep. L.V.V. Sur. 46, 1960, 26 pp. mimeogr.

Schakels NA 3I, 1960. De Ned. Antillen van maand tot maand, 24 pp., ill. DENNERT.

Schakels NA 33, 1960. Het plantenkleed, 32 pp., ill. - Stoffers.

Schakels $S$ 36, 1959. Suriname, activering van natuurlijke hulpbronnen, 32 pp., ill. - Morpurgo, Schulz, Penders, Jonkers.

Schakels $S 39 / N A$ 32, 1960. De rechtsorde in het Koninkrijk der Nederlanden (de basisregelingen), $192 \mathrm{pp}$. (repr. $\left.S_{31} / N_{A} 26\right)$.

Schakels $S 4 I$, 196r. De 8 distrikten, 40 pp., ill. - JoSEPH, van Gorkom. Schakels $S$ 42, 1961. Suriname bouwt aan zijn toekomst, 28 pp., ill. - RoETHOF.

Schakels $S$ 43, 1961. Kleinere volksgroepen, 32 pp., ill. - AdHIN, SAMSON, LOOR, STREEFLAND.

Schakels $S$ 44, 1961. Suriname in kaart en foto, 23 pp. $20 \frac{1}{2} \times 29 \mathrm{~cm}, \mathrm{I}+$ 12 maps, 8 phot. - ZONNEVELD.

Schakels S 45, 1961. Wetenschappelijk onderzoek van het binnenland, 36 pp., ill. - GEIJSKES, JONKER.

Schiel, Ruth: Onzichtbare vleugels. Vertaald door M. A. M. RenesBoldingh. Uitgeverij De Graafschap, Aalten, (1952), 208 pp.

Translation of: Flügel des Charadius, Rainer Wunderlich Verlag, Hermann Leins, Tübingen, 1950. - Novel, dealing with the life of Maria Hartmann, belonging to the Moravian Brethern in Surinam, who died 30. XII. 1853 .

(Schouten, G. J.) 'see: An essay on journalism and freedom of the press. Second Caribbean Geological Conference, Programme, Mayagüez, Puerto Rico, 4-9. I. 1959, College of Agriculture, Univ. of Puerto Rico, 46 pp. $20 \frac{1}{2} \times 13 \frac{1}{2} \mathrm{~cm}$ photopr., fig. 
Semanario. Redactor: Donald LAclé. Impreso: General Printing Co., Aruba. Año $I$ - Nr. I, I5. IX. 1960, 20 pp., $28 \times 21 \frac{1}{2} \mathrm{~cm}$., ill., cover excl.; 2, 23. IX.; 3, 1. X.; 4, 8. X.; 5, 15. X.; 6, 22. X. 1960; etc. [New Aruban weekly.]

Simons, R. D. G. Рн.: Rapport over de noodzakelijkheid van de oprichting van een Tropisch Instituut in Suriname. (1950), $9+3$ pp. $33 \times 21 \mathrm{~cm}$ mimeogr., folding map, col. fig. on cover.

SKS AMS tien jaar. Paramaribo 1960, 19 pp. $27 \frac{1}{2} \times 21 \mathrm{~cm}$, ill.

Small, C. V. J.: Bacoven studiereis. Meded. Landbouwproefsta. Suriname 28, 1961, viii + 57 pp., 45 figs. excl.

Sovan, C. A.: Waar Moehammad bekeert. Een antwoord op "Waar Mohamed regeert" door P. M. Légêne... Surin. Islam. Vereen. Paramaribo, (1943), 55 pp. 2 I $\times$ I $3 \frac{1}{2} \mathrm{~cm}$.

Statistiek van de meteorologische waarnemingen in de Nederlandse Antillen. Statistics of the meteorological observations in the Netherlands Antilles. 1957, 5e Jrg., (1958), 24 pp. $21 \times 28 \mathrm{~cm}$ mimeogr., 3 pls. excl. $-195^{8}$, 6e Jrg., (1959), 31 pp., 3 pls. - 1959, 7e Jrg., (1960), 35 pp., 3 pls. -1960 , 8e Jrg., (196r), 37 pp., 4 pls. - I957-1959 Dept. Econ. Zaken, Bureau voor de Statistiek; 1960 Statistiek en Planbureau.

Statistische Mededelingen. Dienst Econ. Zaken en Welvaartszorg, Bureau voor de Statistiek, Ned. Antillen, about $40 \mathrm{pp}$. each $21 \times 28 \mathrm{~cm}$. 1957: 4e Jaargang No. 12, Juni 1957 - 5e Jrg. 4, Oct.; 1958: 5e Jrg. 5, Nov. 6e Jrg. 4, Okt.; 1959: 6e Jrg. 5, Nov. - 7e Jrg. 4, Okt.; 1960: 7e Jrg. 5, Nov. -8 e Jrg. 4, Okt.; 1961: 8e Jrg. 5, Nov. - 9, Maart. - Statistische Mededelingen Nederlandse Antillen, Statistiek- en Planbureau (since

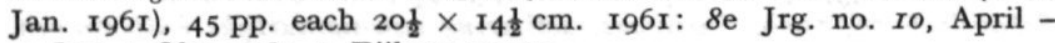
ge Jrg. 4, Okt. r96I + Bijlage, i i pp.

Statistisch Jaarboek 1959. Nederlandse Antillen. Dep. Econ. Zaken Welv. Bureau voor de Statistiek, (Curaçao, 1960), viii + 68 pp. Statistisch Jaarboek 196o. Nederlandse Antillen. Statistiek- en Planbureau, (r96r), viii + 68 pp. - Statistical Yearbook. Anuario Estadístico.

Sticusa. Nederlandse Stichting voor Culturele Samenwerking met Suriname en de Nederlandse Antillen. Amsterdam, 1960, 34 pp. $20 \frac{1}{2} \times 14$ $\mathrm{cm}$. [Program for 1960.]

Stockley, G. M.: The geology of British Guiana and the development of its mineral resources. Bull. Brit. Guiana Geol. Survey 25, 1955, 102 pp., 5 pls. excl., 2 folding maps, 5 loosely inserted maps in pocket at back cover.

Stoffers, A. L.: De Nederlandse Antillen. Het plantenkleed. Schakels NA 33, 1960, 32 pp., 17 figs., cover fig. excl.

Studies on the fauna of Curacao and other Caribbean Islands, edited by P. Wagenaar Hummelinck. Publ. Found. Sci. Research Surinam Neth. Antilles; Nijhoff, The Hague.

Volume $X$, Publ. 21, Feb. 1960, viii + I57 pp., 37 figs., 6 plates excl. Contributions by Boeseman (fishes), DU BoIs - REymond Marcus (tardigrades, worms), Buck (beetles), HAAs (molluscs), HooIJER (mammals), Husson (rodents), LINDER (crustaceans) and MARcus (flatworms). 
Volume $X I$, Publ. 22, Dec. 1960, viii +97 pp., 89 figs.

Contributions by CoBben, Drake \& CoBben and Wygodzinsky on 'The Heteroptera of the Netherlands Antilles'.

Volume $X I I$, Publ. 23, Dec. I961, viii +373 pp., Ior figs., 27 plates \& frontispice excl. [Jan. I962].

BAYER on 'The shallow-water Octocorallia of the West Indian Region'.

Studies on the fauna of Suriname and other Guyanas, edited by D. C. Geijskes \& P. Wagenaar Hummelinck, vol. $I I I$. Publ. Found. Sci. Res. Surinam Neth. Ant. 20, 1959, viii + 191 pp., 76 figs., 9 plates excl. Contributions by BAYER (octocorals), BRUIJNING (mosquitoes), GeiJskes (dragonflies), Hoedeman (fishes) and Holthuis (crustaceans).

Suara - IVC, Officieel orgaan van de Indische Vereniging Curaçao. re jaargang No. 6, Juli 1961, 7 pp. $33 \times 22 \mathrm{~cm}$. mimeogr.

Suchtelen, N. J. van: Verslag van de VIII Inter-American Cacao Conference. Versl. rapp. Dep. L.V.V. Sur. 43, 1960, 21 pp. mimeogr.

Surinam and the Netherlands Antilles. From dependency to partnership. (1955), 36 pp. $20 \times 14 \mathrm{~cm}, 6$ phot.

Suriname en de Nederlandse Antillen, see: Alle Hens.

Suriname. Documentair overzicht verstrekt door het Bureau Algemene Voorlichting van het Kon. Instituut voor de Tropen, Amsterdam, 1960, 28 pp. $25 \times 15 \mathrm{~cm}$.

Suriname. Handelsstatistiek I946. Staat, houdende overzicht van den inen uitvoer van goederen. Algemeen Bureau voor de Statistiek, (Paramaribo), Juni 1947, 200 ex., (89) pp. $27 \times 21 \mathrm{~cm}$. -1947 , Staat,... de in- en uitvoer..., Juli 1948, (92) pp. - r948, 1949, (97) pp. - 1949, 1950, (III) pp. I950, Juni 1951, 250 ex., (II3) pp. - I95I, Mei 1952, 275 ex., (II8) pp. 1952, Juli 1953, 300 ex., (124 + 4) pp.

Suriname in cijfers. Surinam in figures. Algemeen Bureau voor de Statistiek; Centraal Archief, Departement van Binnenlandse Zaken, Paramaribo; $29 \times 21 \mathrm{~cm}$. I, Income distribution 1950 , 1954. - 2, Imports and exports 1953, 1954. - 3, Motor vehicles statistics; first haltyear 1954, 1954. - 4, Imports and exports 1954, 1955. - 5, Imports and exports 1955, 1956, IoI pp. - 6, Imports and exports 1956, 1957, 106 pp. -7, Motor vehicles statistics second half year $1956,1957,31 \mathrm{pp}$. mimeogr. -8 , Motor vehicles statistics second halfyear 1957, 1958, 34 pp. - 9, Imports and exports 1957 , 1958. - Io, Statistics of general education 1957, 1959, 52 pp. - II, Imports and exports 1958, 1959, 192 pp. - I2, Statistics of general education 1958, 1960, 54 pp. - I3, Income distribution, 1960, 37 pp. mimeogr.

Suriname in kaart en foto, see: ZONNEVELD.

Suriname nummer, see: Rostra Economica.

Suriname. Operatie "Sprinkhaan". Operatie "Schildpad". Dept. Opbouw Suriname, (1960), vii +12 pp. $20 \times 14 \frac{1}{2} \mathrm{~cm}$ mimeogr., 2 maps excl.

TAYLOR, WILLIAM RANDOLPH: Marine algae of the eastern tropical and subtropical coasts of the Americas. Ann Arbor, The University of Michigan Press, 1960, xii +870 pp., I4 figs., 80 plates. 
Telefoongids voor het eiland Aruba. Telefoondienst Aruba, September 1959, xxviii + I4I +2 I + I2 Pp.

Telephone Directory Lago January I, r96o. Lago Oil \& Transport Co., Ltd., Aruba, 87 pp. $21 \frac{1}{2} \times 14 \frac{1}{2} \mathrm{~cm}$.

Terlingen, JuAn: Lengua y literatura españolas en las Antillas Neerlandesas. I. Las Antillas Neerlandesas en su vecindad. Una edición del Ministerio de Asuntos Culturales de las Antillas Neerlandesas y el Servicio de Información del Gobierno de las Antillas Neerlandesas. - Originalmente publicada en forma algo diferente en 'Boletin de Filologia', publicación del Instituto de Filología de la Universidad de Chile. 39 pp. $19 \times 1$ r cm.

The Caribbean. The Newsletter of the Caribbean Commission, Hato Rey, Puerto Rico, Vol. I, No. I. May-Nov. 1960, 7 pp.; 2, Dec. 196o; 3, Jan. 196I (publ. Dec. 1960) - Io, Aug. 196I; 80 pp. $28 \times 2$ I $\frac{1}{2} \mathrm{~cm}$ photopr., ill.

The Caribbean. The Newsletter of the Caribbean Organization. Hato Rey, Puerto Rico, Vol. I, No. I, Sep. - Oct. I96I, p. I-I2, (Special inauguration issue); 2, Nov.-Dec. I961, p. 13-22; 3, Jan. 1962, p. $23-30,28 \times 21 \frac{1}{2} \mathrm{~cm}$ photopr., ill.

The Caribbean Journal of Science. Institute of Caribbean Studies, University of Puerto Rico, Mayagüez [Quarterly]; Vol. I, no. I - Feb. I96r, 38 pp., $28 \times 2 \mathrm{I} \frac{1}{2} \mathrm{~cm}$ mimeogr.

The Roar. Aruba Lions Club, Aruba, September I96r, I6 pp. excl. cover, ill. $24 \times 15 \frac{1}{2} \mathrm{~cm}$.

ThIEL, P. H. van: De malariaproblematiek in verband met de uitvoering van het Brokopondo-project. Stichting Surinaams-Nederlands Instituut voor de Volksgezondheid in Suriname [Sunevo], 's-Gravenhage, (196r), iv +83 pp. $34 \times 21 \mathrm{~cm}$ mimeogr., 5 figs, 5 maps excl.

Tivoli Jubileum Gazet I92I-I96I. Aruba, 1961, 32 pp. $23 \frac{1}{2} \times 15 \frac{1}{2} \mathrm{~cm}$, ill. TJon-A-Ten, E. R.: A study of Cooperatives in Jamaica and Puerto-Rico. Versl. rapp. Dep. L.V.V. Sur. 5I, I96I, 53 pp.

TJon Sien Kie, ANdré Philibert Jien Sieuw: Icterus neonatorum simplex. Een literatuur- en proefondervindelijk onderzoek. (A study of literature and experimental research on icterus neonatorum simplex.) Diss. Amsterdam 9. XI. I96I, Ioo pp., 9 figs.

UBELS, E.: Modernisering van de rijstbouw in Nickerie. Meded. Landbouwproefsta. Sur. 26, I96I, iv + 59 pp., II figs. incl. cover.

Veldkamp, J.: Zwaartekracht in Suriname. Wetensch. Rapport W. R. 57-or I, K.N.M.I., De Bilt, 1957, ii +13 pp. $30 \times 21 \mathrm{~cm}$ mimeogr.

Verhoeven, J. M.: Jaarverslag I96o Stichting Wetenschappelijke Bibliotheek. Willemstad, (196I), I4 pp. 2 I $\times$ I4 cm mimeogr.

Verkade, Eline: De Kankantrie. Bekroond toneelspel in drie bedrijven. Toneeluitgeverijen Maestro \& Maestro Junior (A. J. G. Strengholt's Uitg.), Amsterdam, 196r, 70 pp.

Vereniging Bedrijfscontact Nederland-Suriname. Verslag over het achtste verenigingsjaar... 1957. ('s-Gravenhage, 1958), (37) pp. $27 \times 21 \mathrm{~cm}$ mimeogr. - Verslag over het negende verenigingsjaar... 1958, (1959), (35) pp. 
Vereniging Surinaams Bedrijtsleven. Verslag over het zesde verenigingsjaar 1955. Paramaribo, (1956), 60 pp. $23 \frac{1}{2} \times 16 \frac{1}{2} \mathrm{~cm}$. - Verslag over het zevende verenigingsjaar, 1956 (1957), 70 pp. - Verslag. . . achtste... 1957, (1958), 79 pp. - Verslag... negende... 1958, (1959), 88 pp.

Verslagen en rapporten van het Departement van Landbouw, Veeteelt en Visserij in Suriname, $29 \times 20 \frac{1}{2} \mathrm{~cm}$ mimeogr. (Ministerie van Landbouw etc. since No. 52 )

40, I959, TER Horst, Nota garantieprijs voor pinda; 4I, 1960, RELYveld, Orientatie-reis landbouw-onderwijs; 42, Appelman \& Dirven, Melkveeproefbedrijf; 43, van Suchtelen, Cacao Conferentie; 44, DEI Prado, Meeting on Cooperatives; 45, KUIL, Teken bij rundvee; 46 , SAmson, Impressies; 47, I96I, DEL Prado, Advisory Committee; 48. Hooghiemstra, Mond- en klauwzeer; $5 I$, TJon-A-Ten, Cooperatives; 52, KUIL, Kunstmatige inseminatie; 53, KUIL, Uierontsteking 54, Lionarons, Visserij in Brits Guyana.

Verslag van de Commissie tot bestudering van staatkundige hervormingen in Suriname. Deel I. Documentaire inleiding. Staatsdrukkerij- en Uitgeverijbedrijf, 's-Gravenhage, 1948, I44 pp., 6 figs.

Verslag van de toestand van het Eilandgebied Aruba over 1959. (Aruba, I960), xii + I04 pp. $29 \frac{1}{2} \times 21 \mathrm{~cm}$ mimeogr. - Verslag... over 1960. (Aruba, 196r), xiv + ro3 pp.

Verslag van de toestand van het Eilandgebied Bonaire van het jaar I96o. (Bonaire, 196r), i +80 pp. $29 \frac{1}{2} \times 21 \mathrm{~cm}$ mimeogr., table excl.

Verslag van het Departement van Onderwijs en Volksontwikkeling over de jaren 1950 en 195 r. Paramaribo, (1952), 63 pp., ill., 3 folding tables.

Vicioso, José Ramón: Dioramas (Poesias). Segunda edición. Tipografía La Nación, Caracas, 1946, 63 (69) pp.

Vicioso, José Ramón: Isla sin bosques. Ilustraciones de JoHN PANDELlIS. Printed by Aruba Drukkerij N.V., (1960), 56 pp. $16 \frac{1}{2} \times 10 \frac{1}{2} \mathrm{~cm}$. [Poetry.]

Vicioso, José Ramón : Páginas Arubanas. Tipografía La Nación, Caracas, I946, I52 pp.

WagenaAr Hummelinck, P. \& Tacoma, J.: Studies on the physical anthropology of the Netherlands Antilles. I \& II. Uitgaven Natuurwet. Werkgroep Ned. Antillen, No. 9, 1959, 36 pp., ill. - From W. I. Gids 39.

Walterus, FR. M. \& JANSEN, H. J.: Nos Tera, deel III. Ons Koninkrijk en de werelddelen. R.K. Boekhandel 'St. Augustinus', Curaçao, 1960, II 7 pp., ill.

Werk-Conferentie Landbouw-Voorlichting. 25-19 mei 1959. Uitgave Dienst Landbouw, Paramaribo 1959, 32 pp., cover, ill.

Wetenschappelijke Bibliotheek, Jaarverslag 1959, see: CoOMANs; 1960 , see: VERHOEVEN.

WESTENDORP BOERMA, J. J.: Een geestdriftig Nederlander, Johannes van den Bosch. N.V. Em. Querido's Uitgeversmij Amsterdam, 1950, 199 pp., portr. excl. 
Wij in de Antillen. Weekblad voor het gezin. Uitgeverij 'Antilliaanse Periodieken'. Drukker: 'Ediciones Populares'. Redakteur Z. J. SPRong. Curaçao. Ie Jrg. Nr. I, Io november I960, 36 pp. ill., cover excl. 2500 copies printed. $-2, \mathrm{I} 7 . \mathrm{XI}-5,8 . \mathrm{XII}, 36 \mathrm{pp}$. each; 6 , I $5 . \mathrm{XII}-8$, 29.XII.I960, 32 pp. each; 9, 19.I - 24, 4.V.r196I, 32 pp. each. - Discontinued.

Wikor (special number: Suriname en de Nederlandse Antillen), 7, nr. 3, March 1959, p. 67-106, $19 \times 13 \mathrm{~cm}$, ill., 8 pls. \& cover ill. excl.

Wilhelmy, Herbert: Klimamorphologie der Massengesteine. Georg Westermann Verlag, Braunschweig, I958, $23^{8}$ pp., I37 figs.

Aruba, p. 10, 88-89, 92, 103, I37-144, 148-149, 151-152, 200; fig. 57 , 97-104.

Wiт, TH. P. M. DE: The Wageningen rice project in Surinam. A study on the development of a mechanized rice farming project in the wet tropics. Diss. Wageningen, 9. XII. I960, 293 pp., 20 figs., 64 phot. excl.

Woordenlijst van het Sranan-tongo. Glossary of the Suriname Vernacular. Bureau Volkslectuur, N.V. Varekamp \& Co, Paramaribo, r96r, xv + IO2 $\mathrm{pp}$.

"Deze woordenlijst werd samengesteld in nauwe samenwerking met de door het Gouvernement van Suriname in januari r96o ingestelde spellingscommissie voor het Sranantongo. Deze commissie bestaat uit de heren: pater A. Donicie, drs. Ch. H. Eersel, W. A. Leeuwin, Lou Lichtveld (voorzitter), N. Oedraysing Varma en dr J. Voorhoeve (secretaris)."

Wosuna I954-I959. Netherlands Foundation for the Advancement of Research in Surinam and the Netherlands Antilles Wosuna, (Sept.) 1960, Amsterdam, 32 pp. $21 \frac{1}{2} \times I_{5} \mathrm{~cm}$, fig., 7 phot. on 4 pls. \& cover excl.

Zeekaarten Ned. Antillen, Hydrografisch Bureau [obtainable at Fa. Gebr. van Cleef. 's-Gravenhage]: Curaçao, Bonaire en Aruba I : 300.000 , 1954, 2223, $99 \times 69 \mathrm{~cm}$. - St. Maarten, Saba, St. Eustatius en omliggende eilanden I : $250.000,1956,2210,94 \frac{1}{2} \times 82 \frac{1}{2} \mathrm{~cm}$. - Plannen Bovenwindse Eilanden; St. Maarten, Saba, St. Eustatius I : 75.000; Oranjebaai, Filipsburg I : 15.000; 1960, 2716, 71 ×52 cm. - Plannen Curaçao en Aruba, 1960, I715; Paardenbaai I : 15.000; Caracas Baai, Spaansche Water en Fuik Baai $\mathbf{I}$ : I0.000; Sint Nicolaas Haven $\mathbf{I}$ : ro.ooo; Bullen Baai I : 7.500; $104 \times 71 \mathrm{~cm}$. - Curaçao I : 75.000, 1960, $2213,104 \times$ $71 \mathrm{~cm}$. - Aruba I :50.000, 1960, 22II, $104 \times 71 \mathrm{~cm}$; Commandeursbaai I : 15.00o. - Curaçao, St. Anna Baai en Schottegat I : 7.500, 1958, 2714 , $7 \mathrm{r} \times 64 \mathrm{~cm}$.

Zeemansgids voor Suriname en de Nederlandse Antillen. Derde druk, 1953. Ministerie van Marine, Afdeling Hydrografie; Gebr. van Cleef, 's-Gravenhage, xiv +34 pp. $26 \times 18 \mathrm{~cm}, 2$ folding maps excl. - Aanvullingsblad No. 5-1959, ro pp. Lichtenlijst en betonningsstaat 1959, 26 pp.

ZONNEVELd, J. I. S.: Suriname in kaart en foto. Schakels $S$ 44, 1961, 23 pp. $20 \frac{1}{2} \times 29 \mathrm{~cm}$, 12 maps, 8 aerial phot., cover.

P.W.H. 